\title{
He and Si surface inhomogeneities of four Bp variable stars ${ }^{\star \star \star}$
}

\author{
M. Briquet ${ }^{1,4}$, C. Aerts ${ }^{1}$, T. Lüftinger ${ }^{2}$, P. De Cat ${ }^{1, \star \star \star}$, N. E. Piskunov ${ }^{3}$, and R. Scuflaire ${ }^{4}$ \\ ${ }^{1}$ Instituut voor Sterrenkunde, Katholieke Universiteit Leuven, Celestijnenlaan 200 B, 3001 Leuven, Belgium \\ 2 Institut für Astronomie, Universität Wien, Türkenschanzstr. 17, 1180 Wien, Austria \\ 3 Uppsala Astronomical Observatory, Box 515, 751 20, Uppsala, Sweden \\ ${ }^{4}$ Institut d'Astrophysique et de Géophysique de Liège, Université de Liège, allée du Six Août 17, 4000 Liège, Belgium
}

Received 26 May 2003 / Accepted 4 September 2003

\begin{abstract}
We present ground-based multi-colour Geneva photometry and high-resolution spectra of four variable B-type stars: HD 105382, HD 131120, HD 138769 and HD 55522. All sets of data reveal monoperiodic stars. A comparison of moment variations of two spectral lines, one silicon line and one helium line, allows us to exclude the pulsation model as being the cause of the observed variability of the four stars. We therefore delete the four stars from the list of candidate slowly pulsating B stars. We attribute the line-profile variations to non-homogeneous distributions of elements on the stellar surface and we derive abundance maps for both elements on the stellar surface by means of the Doppler Imaging technique. We confirm HD 131120 to be a He-weak star and we classify HD 105382, HD 138769 as new He-weak stars. HD 55522 has the solar helium abundance but the mean abundance value of He varies by 0.8 dex during the stellar rotation. For HD 131120 and HD 105382, helium is enhanced in regions of the stellar surface where silicon is depleted and depleted in regions where silicon is enhanced.
\end{abstract}

Key words. line: profiles - stars: individual: HD 131120, HD 105382, HD 138769, HD 55522

\section{Introduction}

Aerts et al. (1999) and Mathias et al. (2001) started a long-term spectroscopic project to monitor some 20 candidate slowly pulsating B stars (SPBs) selected among many candidates discovered from the HIPPARCOS mission (Waelkens et al. 1998). In this selected sample, four stars are distinguishable from confirmed SPBs (De Cat 2001) although they showed line-profile variations similar to those of SPBs at first sight: HD 105382 (B 2 III), HD 131120 (B 2.5 III), HD 138769 (B 3 IVp) and HD 55522 (B 2 IV/V).

In Briquet et al. (2001a) we have already made an extensive study of the star HD 105382 but we could not choose between rival hypotheses, namely non-radial pulsation or rotational modulation, to explain the observed monoperiodic variability of the star. In Briquet et al. (2001b) a comparison of the moment variations of silicon lines to the ones of a helium line allowed us to exclude the pulsation model for the star HD 131120. In this paper, we show that this way to discriminate between stellar pulsation and stellar spots can be used for HD 105382 and the two other B-type stars HD 138769 and HD 55522 so that we attribute the variations of the four stars to

Send offprint requests to: M. Briquet,

e-mail: maryline@ster.kuleuven.ac.be

* Based on observations obtained with the Swiss photometric telescope and ESO's CAT/CES telescope, both situated at La Silla, Chile.

$\star \star$ Appendix A, Tables 1 and 2 and Figs. 9, 11, 13 are only available in electronic form at http://www. edpsciences.org

$\star \star \star$ Postdoctoral Fellow of the Fund for Scientific Research, Flanders. rotational modulation instead of stellar pulsation. Moreover we derive abundance maps for both elements $\mathrm{Si}$ and $\mathrm{He}$ on the stellar surface of these four studied stars by means of the Doppler Imaging technique.

The paper is organized as follows. In Sect. 2 we describe the observations that we have at our disposal and we derive some physical parameters of the stars. In Sect. 3 we show the outcome of the frequency analysis on photometric and spectroscopic data together with the variations of the moments of the $\mathrm{Si}$ and $\mathrm{He}$ lines. Abundance distributions on the stellar surface of both elements are derived in Sect. 4. Conclusions are given in Sect. 5.

\section{Data and physical parameters}

We have three datasets at our disposal: HIPPARCOS photometry, multicolour Geneva photometry and high-resolution spectroscopic data. The Geneva photometric observations were obtained with the Swiss telescope situated at La Silla in Chile. The spectroscopic data were obtained with the CAT/CES telescope of ESO in Chile. The spectral domain was $[4115,4135] \AA$ in order to get the Si II-doublet with lines at $\lambda \lambda 4128,4130 \AA$ so that we also have the He I $4121 \AA$ line. A log of the observations is given in Table 1 . The number of observations and the ranges of their Julian Dates are given in Table 2 for the spectroscopy. For a complete description of the observations and data reductions we refer to Aerts et al. (1999) and De Cat (2001). 
Table 3. Physical parameters of the four studied stars.

\begin{tabular}{ccccc}
\hline \hline & HD 131120 & HD 105382 & HD 138769 & HD 55522 \\
\hline$T_{\text {eff }}(\mathrm{K})$ & $18250 \pm 420$ & $17400 \pm 400$ & $17500 \pm 400$ & $17400 \pm 400$ \\
$\log g$ & $4.10 \pm 0.15$ & $4.18 \pm 0.15$ & $4.22 \pm 0.15$ & $4.15 \pm 0.15$ \\
$\log L / L_{\odot}$ & $3.13 \pm 0.15$ & $2.89 \pm 0.15$ & $2.98 \pm 0.15$ & $2.95 \pm 0.15$ \\
$M\left(M_{\odot}\right)$ & $6.1 \pm 0.6$ & $5.7 \pm 0.4$ & $5.4 \pm 0.4$ & $5.5 \pm 0.3$ \\
$R\left(R_{\odot}\right)$ & $3.6 \pm 0.8$ & $3.0 \pm 0.6$ & $3.4 \pm 0.6$ & $3.3 \pm 0.6$ \\
\hline
\end{tabular}

A first estimate of the effective temperature and of the gravity of our targets is obtained by means of the photometric calibration by Künzli et al. (1997) to the mean magnitudes in the Geneva filters. The parallax measured by HIPPARCOS provides the distance. This, together with the average visual magnitude, gives the absolute visual magnitude. One obtains the bolometric magnitude and consequently the luminosity taking into account the bolometric correction (BC), which is calculated by means of Flower's relation (1996) between $T_{\text {eff }}$ and $\mathrm{BC}$. With the values for the effective temperature and the luminosity one estimates the mass by using the evolutionary tracks published by Schaller et al. (1992). We also calculated the radius. The results are given in Table 3 . We stress that the standard errors given in Table 3 are formal errors, i.e. errors derived from uncertainties due to interpolation in Künzli et al.'s grid of standard stars. As we are dealing with spotted stars, these formal errors are underestimates of the true uncertainties.

\section{Frequency analysis and moment variations}

For the star HD 131120, our frequency analysis on HIPPARCOS and Geneva photometry and our analysis of the moment variations $\langle v\rangle,\left\langle v^{2}\right\rangle$ and $\left\langle v^{3}\right\rangle$ (for a definition, see Aerts et al. 1992) of both lines of Si and He are fully described in Briquet et al. (2001b). In this section, we describe similar analyses for our three other considered stars.

The frequency analysis on the datasets was performed using the PDM method (Stellingwerf 1978), the Scargle and CLEAN methods (Scargle 1981; Roberts et al. 1987). We tested frequencies from 0 to 3 cycles per day $\left(\mathrm{c} \mathrm{d}^{-1}\right)$ with a frequency step of $0.0001 \mathrm{c} \mathrm{d}^{-1}$ and we searched for multiple periods by prewhitening.

\subsection{HD 105382}

In Briquet et al. (2001a), we performed a frequency analysis for HD 105382 on photometry as well as on high-resolution Si II 4128-4130 A lines. All these data point out that there is only one period of 1.295 days present in the star.

In this paper, we complete our analysis by also computing the first three moments of the He I $4121 \AA$ line in order to compare their temporal variations to those of the Si lines. In $\langle v\rangle$ we found subsequently $2 f$ and $f$, which reduce together the standard deviation by $65 \%$. In $<v^{2}>$ we found subsequently $2 f, f$ and $3 f$. In $\left\langle v^{3}>\right.$ we found $2 f$. Phase diagrams for $\langle v\rangle$, $<v^{2}>$ and $<v^{3}>$ computed from the $\mathrm{Si}$ and He lines are shown in Fig. 1. A comparison of the behaviour of both lines allows us to conclude that the cause of the observed variability of the star cannot be stellar pulsation. Indeed, for a pulsating star, the temporal behaviour and phasing of the moments are the same whatever is the line. Moreover the amplitude of the first moment, which is the radial velocity in the case of pulsation, does not depend too much on the lines for SPBs. Clearly, for HD 105382, the frequency $2 f$ dominates the moments of the He line while it is the frequency $f$ for the Si line. The minimum value for $\langle v\rangle$ and $\left\langle v^{3}>\right.$ of the Si line corresponds to a maximum value for the He line. The peak-to-peak amplitude in $\langle v\rangle$ obtained from the He line is about $20 \mathrm{~km} \mathrm{~s}^{-1}$ while the one obtained from the $\mathrm{Si}$ line is about $10 \mathrm{~km} \mathrm{~s}^{-1}$.

We also show the equivalent width variations of the $\mathrm{Si}$ II $4128 \AA$ line and of the He I $4121 \AA$ line respectively in the left and right upper panels of Fig. 5. The equivalent widths vary with the same frequency as the higher order moments. The relative $E W$ variations of the $\mathrm{Si}$ line and of the He line are about $9 \%$ and $7 \%$ respectively, which is large compared to the ones of confirmed SPBs (De Cat 2001). Moreover De Ridder et al. (2002) have shown that relative $E W$ variations of Si lines never exceed a few percent for slowly-rotating nonradially pulsating $B$ stars.

All these peculiarities of the variations of the line profiles lead us to exclude the pulsational model as an explanation for the variability of HD 105382. As the moment variations of the $\mathrm{Si}$ and He lines resemble those of HD 131120, we also attribute the variability of HD 105382 to rotational modulation. Indeed, for HD 131120, we modelled the line-profile variations by means of a simple model with circular spots for which the flux in the spots differs from that coming from the rest of the star (Briquet et al. 2001b). Such a basic model was able to reproduce the first three moment variations of the lines, the large equivalent width variations as well as a different behaviour of moments of silicon and helium lines. We then concluded that a good explanation for the variability of the star is its rotation in the presence of non-homogeneous distributions of elements on the stellar surface.

\section{2. $H D 138769$}

\subsubsection{The HIPPARCOS and Geneva photometric data}

In the HIPPARCOS measurements all three methods of period determination lead to a clear frequency, which is $f=$ $0.4786 \mathrm{c} \mathrm{d}^{-1}$. This frequency reduces the data standard deviation by $30 \%$. A phase diagram for this frequency is represented in the upper left panel of Fig. 2. Note that the peak-to-peak amplitude of $H_{\mathrm{p}}$-data is only $0.015 \mathrm{mag}$ and the standard deviation of the residuals is some $0.00464 \mathrm{mag}$, which is only slightly larger than the average error on the data of $0.0043 \mathrm{mag}$. We searched for another frequency in the data but we were not 

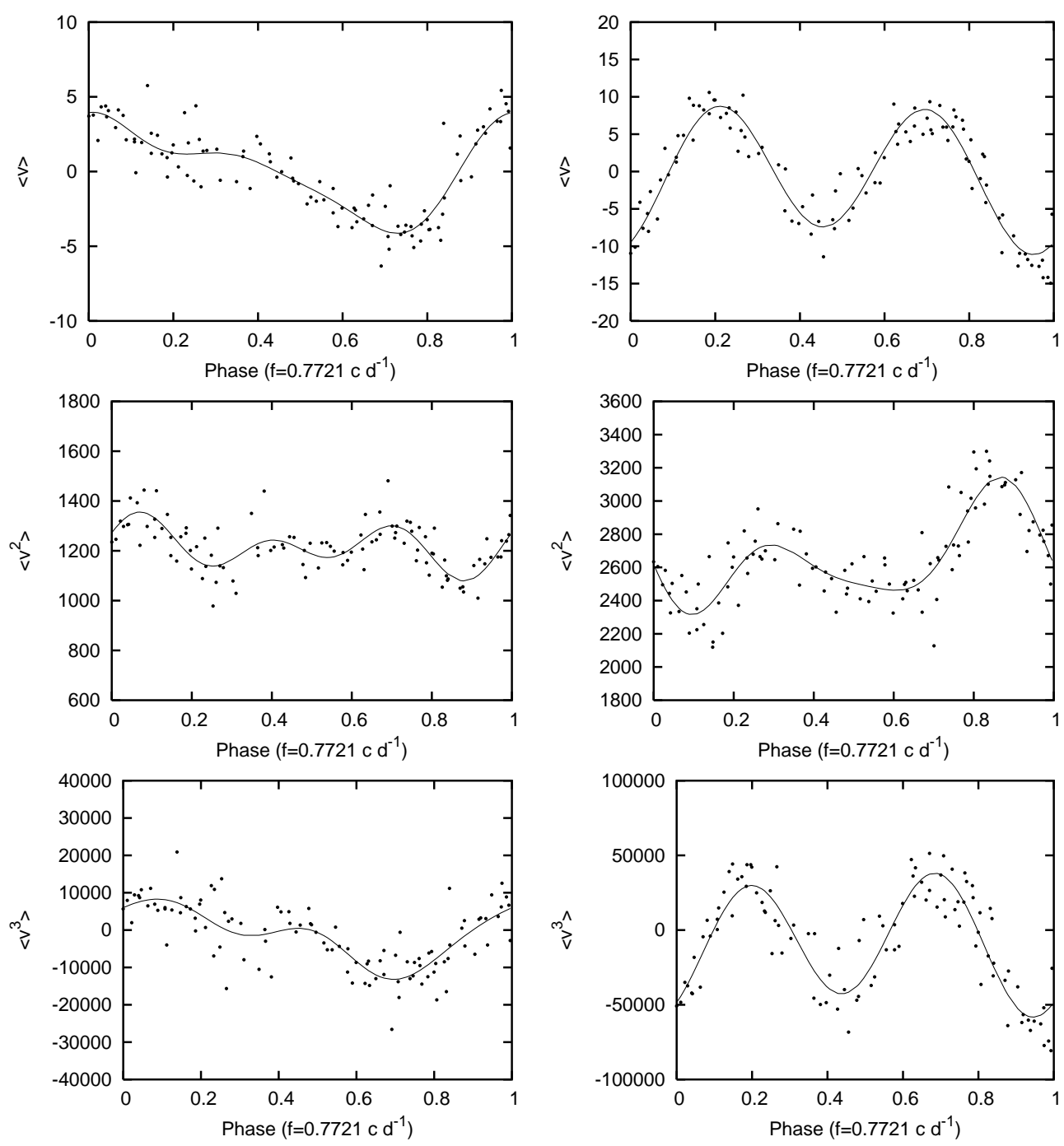

Fig. 1. For HD 105382, first three observed moments of the Si II $4128 \AA$ line (dots, left) and of the He I $4121 \AA$ line (dots, right). $<v>$, $<v^{2}>$ and $<v^{3}>$ are expressed respectively in $\mathrm{km} \mathrm{s}^{-1},\left(\mathrm{~km} \mathrm{~s}^{-1}\right)^{2}$ and $\left(\mathrm{km} \mathrm{s}^{-1}\right)^{3}$. The full lines represent the best harmonic fits for the indicated frequency.

able to determine one. The PDM and Scargle methods led to different candidates.

We found the same main frequency $f$ in the seven filters of Geneva photometry, which reduces the standard deviation by $25 \%$. A phase diagram for the $U$-magnitude is shown in the upper right panel of Fig. 2. As for HIPPARCOS photometry we were not able to derive a second frequency.

\subsubsection{The spectroscopic data}

Through the PDM method we found the frequency $f$ in the first moment computed from the Si II $4128 \AA$ line. After prewhitening with $f$ we found a frequency very close to $2 f$. In $\langle v\rangle$ computed from the He I $4121 \AA$ line, the PDM method leads subsequently to the frequencies $f$ and $2 f$. Using the Scargle method we obtained $1-f$ as first frequency and $2 f$ after prewhitening with $f$. Phase diagrams for $\langle v\rangle$ are shown in the upper part of Fig. 3. The frequency $f$ and its first harmonic reduce the standard deviation of $\langle v\rangle$ computed from the $\mathrm{He}$ line by $74 \%$.
In the second and third moment of the Si II $4128 \AA$ line we were not able to determine a frequency. Concerning the He I $4121 \AA$ line, its second moment does not show a clear frequency while the third moment clearly presents the frequency $f$ and its first harmonic $2 f$. Phase diagrams of these moments of both lines are represented in Fig. 3. The behaviour of $\langle v\rangle$, $<v^{2}>$ and $\left\langle v^{3}>\right.$ resemble the one of HD 131120 (Briquet et al. 2001b). Consequently we conclude that the variability of the star is due to rotational modulation. The equivalent width of both lines varies with $f$ as shown in Fig. 5. The relative equivalent width variation is about $9 \%$ and $17 \%$ for respectively the Si line and the He line.

\section{3. $H D 55522$}

\subsubsection{The HIPPARCOS and Geneva photometric data}

De Cat (2001) already made a period search in photometric datasets of the star HD 55522. In this paper we show again the variations of photometry to underline the different behaviour of 

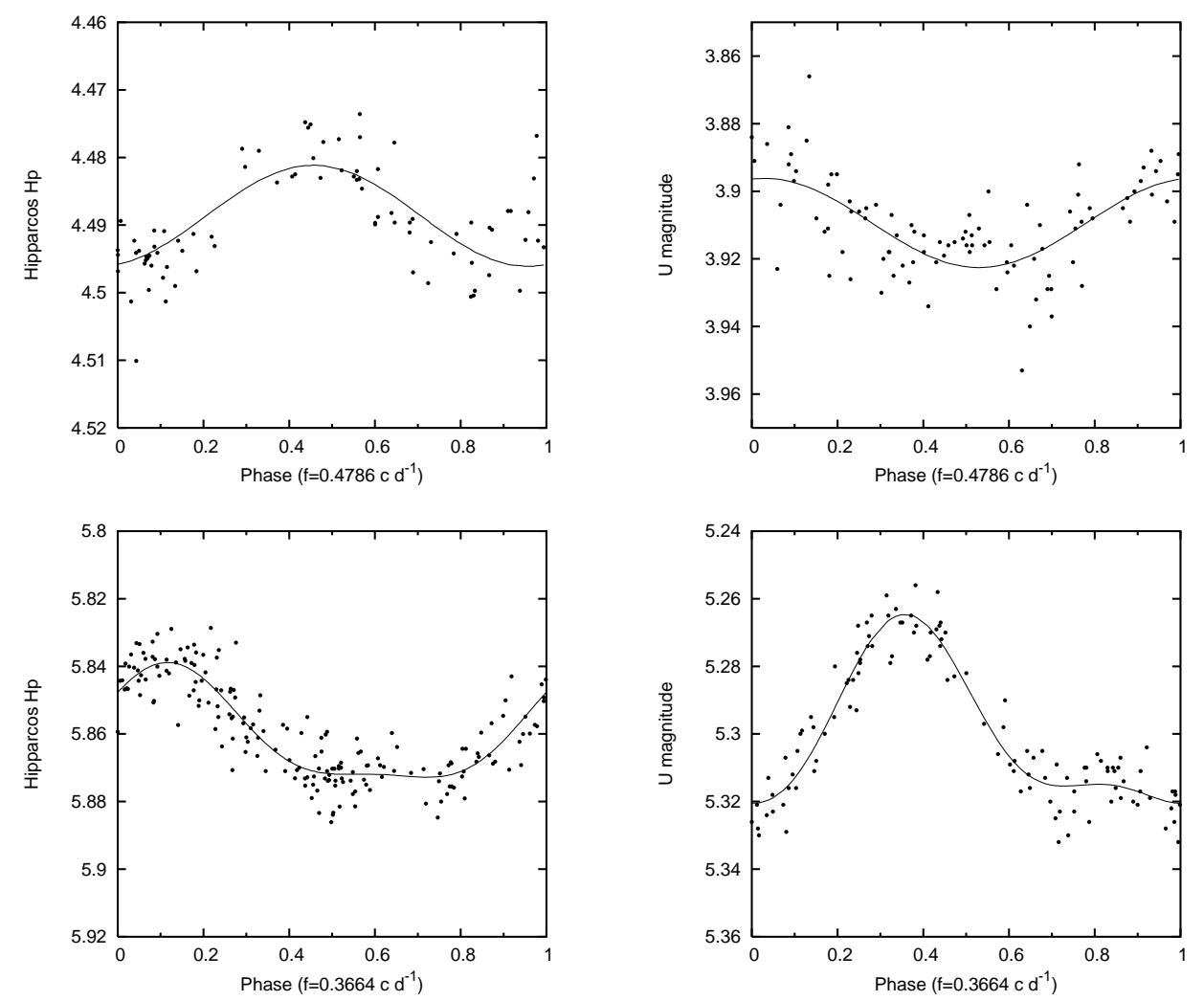

Fig. 2. For HD 138769 (upper) and HD 55522 (lower), phase diagrams of the HIPPARCOS data and of the $U$-magnitude data (dots) are compared with their best sinusoidal fits for the indicated frequency (full lines).

the star compared to confirmed SPBs. For both HIPPARCOS and Geneva photometric data we found subsequently the frequency $f=0.3664 \mathrm{c} \mathrm{d}^{-1}$ and $2 f . f$ with $2 f$ reduce the standard deviation by $53 \%$ and $69 \%$ for HIPPARCOS and Geneva data respectively. Phase diagrams for both types of photometric data are shown in Fig. 2. Clearly the variability of the star is dominated by only one frequency and its first harmonic. No other frequencies can be found in the photometric datasets.

\subsubsection{The spectroscopic data}

The frequency analysis on the Si II 4128-4130 ̊ doublet does not lead to a clear frequency although the frequency $f$ seems to be present in the first moment of the Si II $4128 \AA$ line. However in the first moment of the He I $4121 \AA$ line, the frequency $f$ is clearly found and even its harmonics up to $4 f$. The frequencies $f$ and its first three harmonics reduce the standard deviation of $\langle v\rangle$ by $76 \%$ for the helium line.

In $<v^{2}>$ and $\left\langle v^{3}>\right.$ of the Si II 4128-4130 $\AA$ lines it was not possible to find a frequency. Phase diagrams for $f$ are shown in the left part of Fig. 4. The moments are very noisy and no clear periodicity is observed. For the He I $4121 \AA$ line, however, we found in $\left\langle v^{2}\right\rangle$ the frequency $f$ followed by $3 f$ and in $\left\langle v^{3}\right\rangle$ we found $f$ followed by $2 f$. Phase diagrams of the first three moments of the helium line are shown in the right part of Fig. 4. No other frequency could be found in $\langle v\rangle$ nor $<v^{2}>$ or $\left\langle v^{3}>\right.$. The star is clearly monoperiodic.

As for HD 131120, HD 105382 and HD 138769 we conclude that such typical variations of the moments are not due to pulsation but can be caused by rotational modulation. We recall again their characteristics. Harmonics of the frequency are present in the datasets. The moment variations of the He line are completely different from those of the Si lines. The peak-to-peak amplitude of the first moment computed from the He line is four times larger than the one computed from the Si line.

In Fig. 5 the equivalent width variations of both considered lines are represented for HD 105382, HD 138769 and HD 55522 (those of HD 131120 are given in Briquet et al. 2001b). The ones of the Si line do not show any periodicity and are very noisy while the $E W$ variations of the He line present a clear periodicity with $f$ and harmonics as for the higher order moments. We note that the relative $E W$ variation of the He line is about $35 \%$, which is very large.

\section{Abundance surface mapping}

We interpret the observed monoperiodic variability of the four studied stars to their rotation and inhomogeneities of elements on their stellar surface. The best method available to map surface abundances of CP stars is the Doppler Imaging technique. Thanks to the code INVERS11 developed by Piskunov, we mapped the surface silicon and helium abundance by using the Si II 4128-4130 doublet, the He I $4121 \AA$ line and the He I $4143 \AA$ line. For a detailed description of the code INVERS11, we refer to Appendix A and to Lüftinger et al. (2003). For the spectral synthesis of local line profiles, model atmospheres were calculated with Kurucz's ATLAS9 

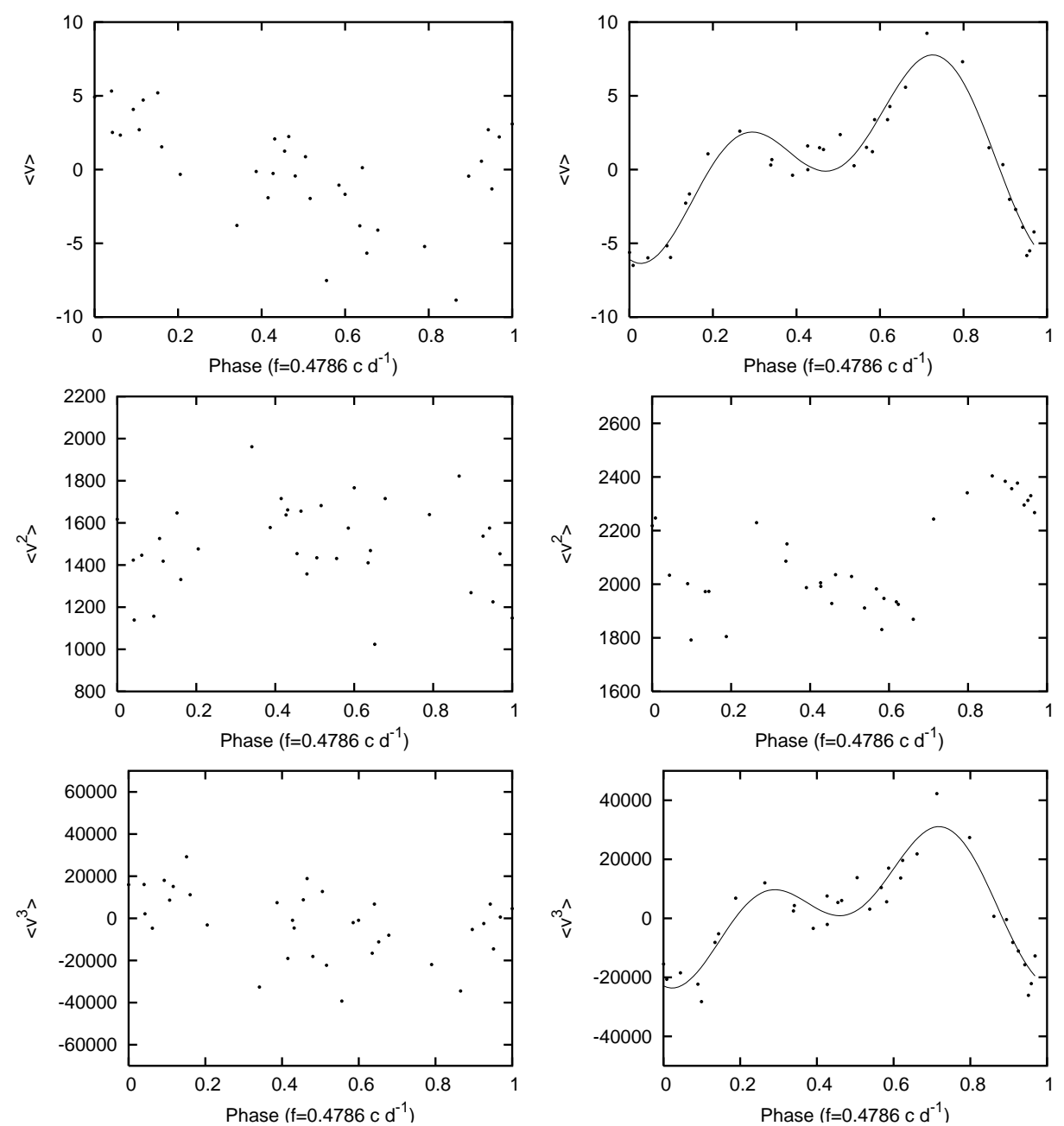

Fig. 3. For HD 138769, first three observed moments of the Si II $4128 \AA$ line (dots, left) and of the He I $4121 \AA$ line (dots, right). $<v>$, $<v^{2}>$ and $<v^{3}>$ are expressed respectively in $\mathrm{km} \mathrm{s}^{-1},\left(\mathrm{~km} \mathrm{~s}^{-1}\right)^{2}$ and $\left(\mathrm{km} \mathrm{s}^{-1}\right)^{3}$. The full lines represent the best harmonic fits for the indicated frequency.

programs (Kurucz 1992, 1993). The Vienna Atomic Line Database (VALD, Piskunov et al. 1995; Ryabchikova et al. 1999; Kupka et al. 1999) provided the atomic line data. Spectra were synthesized with the program SYNTH (Piskunov 1992). We started with solar abundances and adjusted them by comparing observed and calculated spectra.

\section{1. $H D 131120$}

Our mapping of the star HD 131120 was performed in the following way. We varied the parameters $T_{\text {eff }}$ and $\log g$ around their values derived from Geneva photometry. All derived maps showed very similar distributions of elements. We then decided to choose the maps for the $T_{\text {eff }}$ and $\log g$ values found from Geneva data. To illustrate it, the left and right upper part of Fig. 6 correspond respectively to $T_{\text {eff }}=18600 \mathrm{~K}, \log g=4.2$ (values found by de Geus 1989) and $T_{\text {eff }}=18250 \mathrm{~K}, \log g=$ 4.1 (Table 3). The figures are divided as follows:

a- is the mercator projection of the abundance distribution and comprises contour lines around regions of equal abundance; b- shows the mapped stellar surface in four rotational phases $(0.0,0.25,0.5,0.75)$. Below each phase, values for the mean abundance averaged over the visible part can be found. Dark colour means high, bright colour means low abundance.

The projected rotational velocity $v_{\Omega}$ was derived so that theoretical profiles reproduce the observed width of the line. A first estimate for the angle of inclination $i$ was determined from $P_{\text {obs }}=2 \pi R \sin i / v_{\Omega}$ by taking into account the uncertainty on the stellar radius $R$ listed in Table 3. Consequently we varied $v_{\Omega}$ from $55 \mathrm{~km} \mathrm{~s}^{-1}$ to $65 \mathrm{~km} \mathrm{~s}^{-1}$ with a step of $5 \mathrm{~km} \mathrm{~s}^{-1}$ and $i$ from $20^{\circ}$ to $60^{\circ}$ with a step of $10^{\circ}$. We then chose the combination of parameters which minimizes the residuals between observations and the computed line profiles. Note that a difference of $10^{\circ}$ for the inclination does not change very much the outcome, as illustrated in Fig. 6.

The final maps were computed for $T_{\text {eff }}=18250 \mathrm{~K}, \log g=$ 4.1, $v_{\Omega}=60 \mathrm{~km} \mathrm{~s}^{-1}$ and $i=30^{\circ}$. The right upper part of Fig. 6 was derived from the He I $4121 \AA$ line, the left middle part of Fig. 6 from the same line but for $i=40^{\circ}$, the right middle part of Fig. 6 from the He I $4143 \AA$ line, the left lower part of Fig. 6 

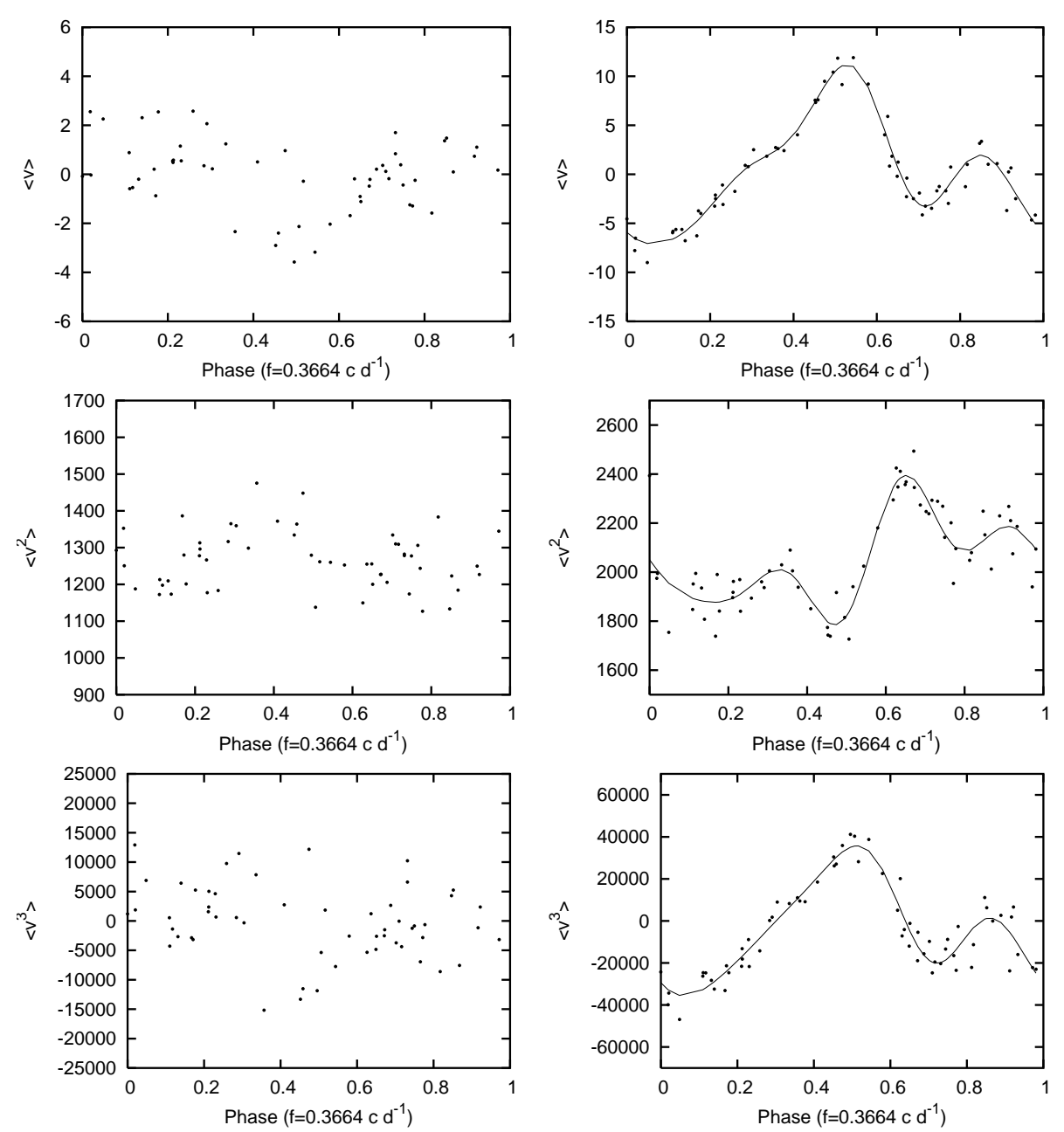

Fig. 4. For HD 55522, first three observed moments of the Si II $4128 \AA$ A line (dots, left) and of the He I $4121 \AA$ line (dots, right). $<v>,<v^{2}>$ and $<v^{3}>$ are expressed respectively in $\mathrm{km} \mathrm{s}^{-1},\left(\mathrm{~km} \mathrm{~s}^{-1}\right)^{2}$ and $\left(\mathrm{km} \mathrm{s}^{-1}\right)^{3}$. The full lines represent the best harmonic fits for the indicated frequency.

from the He I $4121 \AA$ line taking into account the blends of Fe and $\mathrm{O}$ and the right lower part of Fig. 6 from the Si II-doublet with lines at $\lambda \lambda 4128,4130 \AA$. Taking into account blends of other elements evidently gives a better fit to line-profile variations but does not change the structure of the maps. Figure 7 compares the observed and the calculated $\mathrm{He}$ and $\mathrm{Si}$ profiles at different phases of observation from phase zero (top) to the end of the rotation period (bottom).

The right middle and left lower part of Fig. 6 show that helium is globally significantly depleted at the stellar surface relative to solar abundance $(-1.05 \mathrm{dex})$. We find good agreement in both maps in the reproduction of the overabundance spots. A difference between the maps derived for He 4121 and He 4143 can be noted. A more pronounced underabundance centered at the pole (and the less pronounced underabundance around longitude 280) occurs in the He 4143 map. This might be due to the fact that a DI code tends to over- or underinterpret abundances in less visible regions like the pole or sub-observer points, if it does not reach a perfect fit otherwise. Generally abundances in intermediate latitudes are more trustworthy than in extreme regions, especially if there is a mismatch between different lines of the same element. A comparison between, on the one hand the right middle and left lower part of Fig. 6, and on the other hand the right lower part of Fig. 6, shows that helium is enhanced in regions of the stellar surface where silicon is depleted and inversely.

\section{2. $H D 105382$}

In this section we derive abundance surface maps for HD 105382. For HD 131120 we have shown that an uncertainty of a few hundred Kelvin on $T_{\text {eff }}$ and of 0.1 on $\log g$ does not influence significantly the outcome of the Doppler mapping. We therefore fixed the values of these parameters to those found from Geneva photometry. We varied $v_{\Omega}$ from $60 \mathrm{~km} \mathrm{~s}^{-1}$ to $75 \mathrm{~km} \mathrm{~s}^{-1}$ with a step of $5 \mathrm{~km} \mathrm{~s}^{-1}$ and $i$ from $20^{\circ}$ to $60^{\circ}$ with a step of $10^{\circ}$ in order to choose the parameters that minimize the deviation between observations and computed line profiles. As for the previous star, an angle of inclination of the star $i$ that is $10^{\circ}$ lower or higher led to very similar maps. It was then not useful to refine the grid of parameters. The derived maps are shown in Fig. 8 for silicon and helium. Figure 9 compares the observed and the calculated $\mathrm{He}$ and $\mathrm{Si}$ profiles at different phases of observation from phase zero (top) to the end of the rotation period (bottom). 

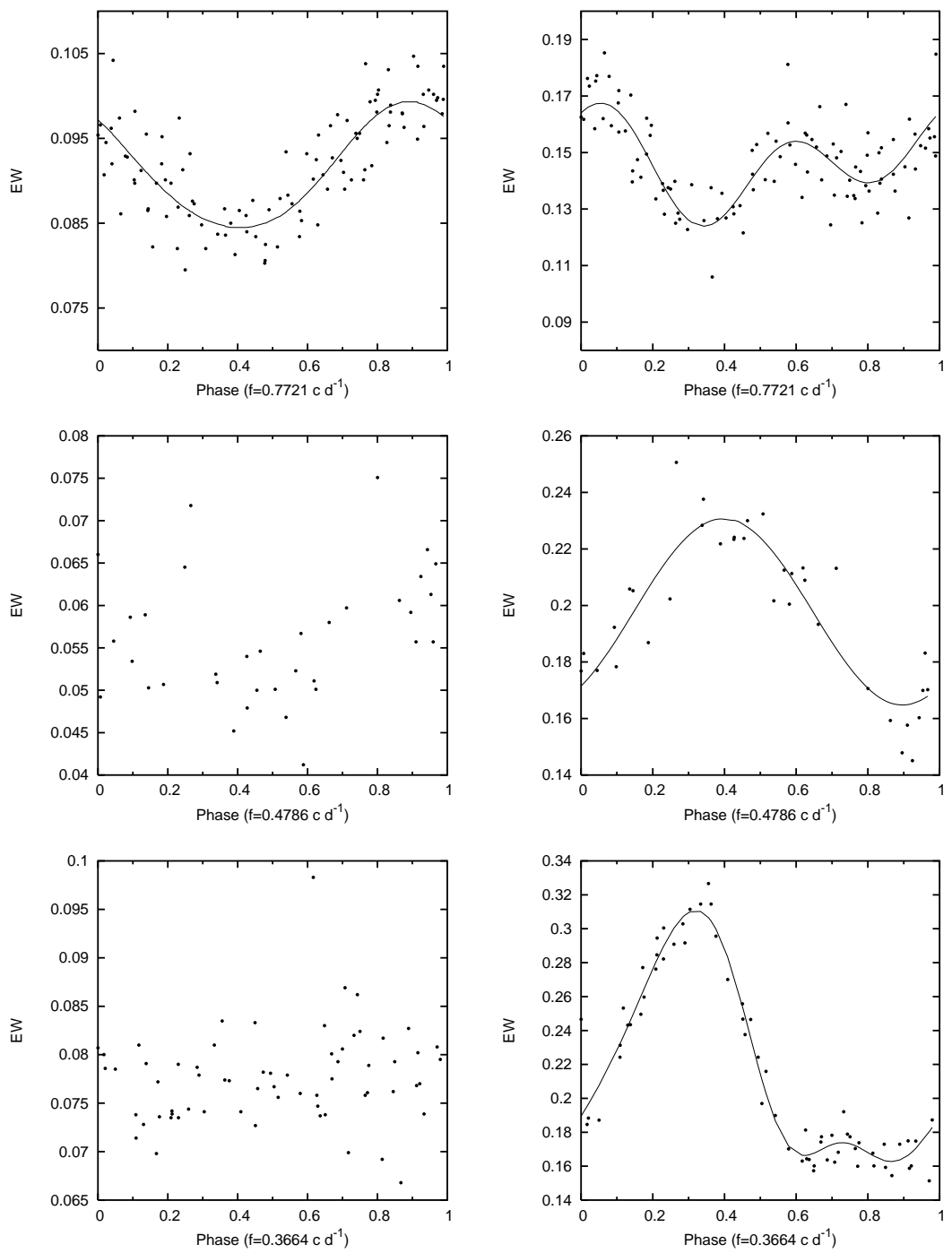

Fig. 5. For HD 105382 (upper), HD 138769 (middle) and HD 55522 (lower), observed equivalent width variations of the Si II $4128 \AA$ A line (dots, left) and of the He I $4121 \AA$ line (dots, right), expressed in $\AA$. The full line is the best fit for the equivalent width.

We conclude the following. Silicon is depleted on average over the stellar surface compared to the Sun $(-4.49$ dex $)$. Helium is significantly depleted on the majority of the stellar surface. On average the stellar surface is very depleted in helium compared to the solar abundance. Two strong spots have a longitude difference of $180^{\circ}$. This corresponds to the two bumps observed in the first moment of the line (see right part of Fig. 1). A less strong third spot is also visible. As for HD 131120 the comparison of maps of both elements shows that roughly where helium is depleted, silicon is enhanced and vice versa. This corresponds to the dephasing observed in the first moment of the lines (see Fig. 1).

\section{3. $H D 138769$}

We derived abundance maps for silicon and helium for HD 138769 by taking $T_{\text {eff }}=17500 \mathrm{~K}$ and $\log g=4.2$ and by varying $v_{\Omega}$ from $60 \mathrm{~km} \mathrm{~s}^{-1}$ to $70 \mathrm{~km} \mathrm{~s}^{-1}$ with a step of $5 \mathrm{~km} \mathrm{~s}^{-1}$ and $i$ from $30^{\circ}$ to $70^{\circ}$ with a step of $10^{\circ}$. The best silicon map was obtained for $v_{\Omega}=70 \mathrm{~km} \mathrm{~s}^{-1}$ and $i=40^{\circ}$ and is shown in Fig. 10. Silicon is clearly underabundant compared to the
Sun. Its observed mean abundance is constant during the rotation of the star. A depleted region is found at the pole while an enhanced spot is situated at the equator. As for HD 131120 (Sect. 4.1), the same remark holds for depleted Si at the pole of HD 138769. We have less confidence in the polar spot, while the appearence of a real overabundant region at the equator is strengthened by an increase in the equivalent width of the spectral lines. For helium the deviation between observed and calculated profiles is very large so that we have less confidence concerning the reliability of the derived map. However it is clear that the star is also a He-weak star since the helium mean abundance is lower than the solar abundance. Figure $11 \mathrm{com}-$ pares the observed and the calculated Si profiles at different phases of observation from phase zero (top) to the end of the rotation period (bottom).

\section{4. $H D 55522$}

We derived abundance maps for silicon and helium for HD 55522 in the same way as for the previous stars. We took $T_{\text {eff }}=17400 \mathrm{~K}$ and $\log g=4.1$. 

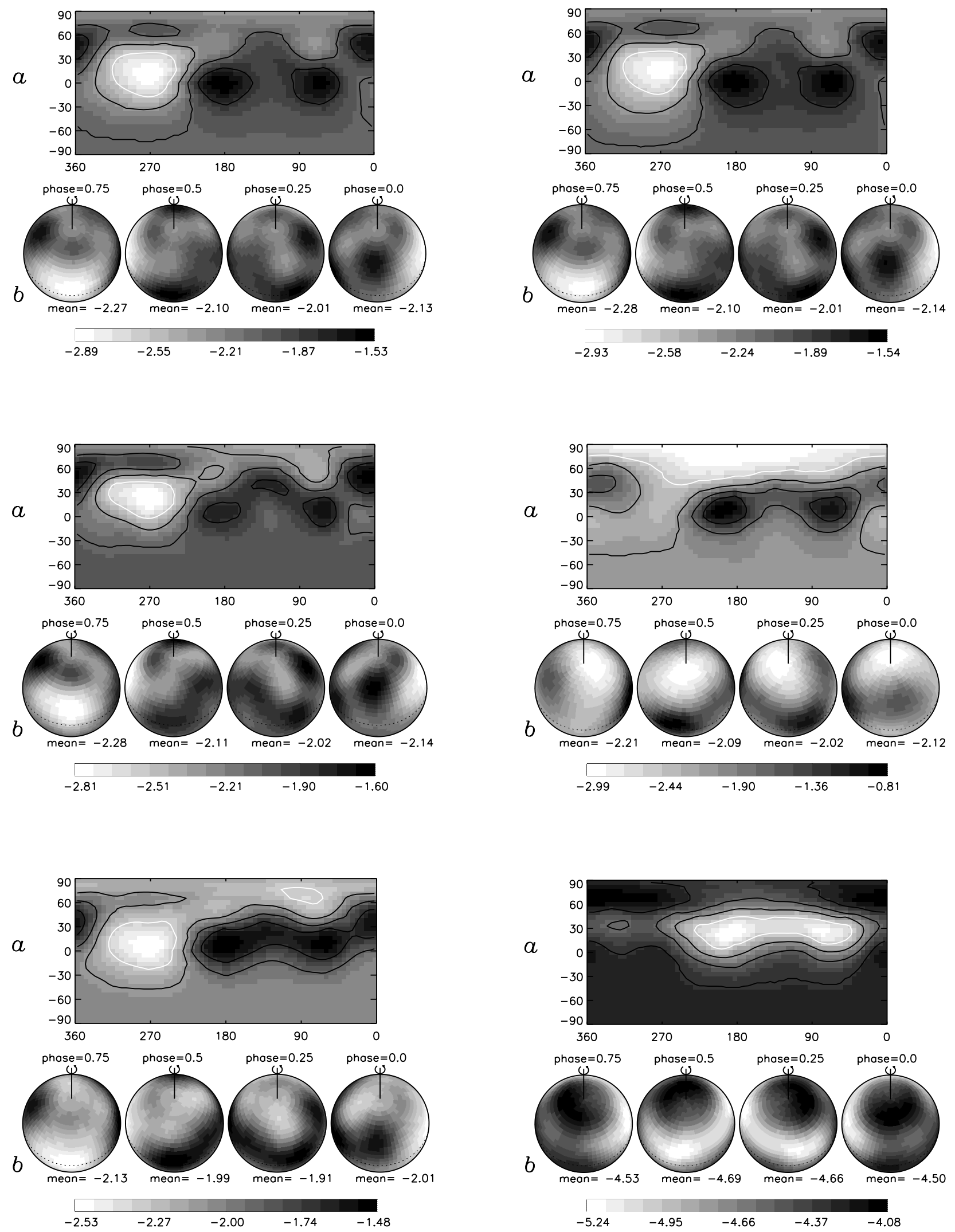

Fig. 6. The abundance distributions on the surface of HD 131120 obtained with INVERS11. Upper left: from the He I $4121 \AA$ line for $T_{\text {eff }}=$ $18600 \mathrm{~K}, \log g=4.2, v_{\Omega}=60 \mathrm{~km} \mathrm{~s}^{-1}$ and $i=30^{\circ}$. Upper right: from the He I $4121 \AA$ line for $T_{\mathrm{eff}}=18250 \mathrm{~K}, \log g=4.1, v_{\Omega}=60 \mathrm{~km} \mathrm{~s}{ }^{-1}$ and $i=30^{\circ}$. Middle left: from the He I $4121 \AA$ line for $T_{\mathrm{eff}}=18250 \mathrm{~K}, \log g=4.1, v_{\Omega}=60 \mathrm{~km} \mathrm{~s}^{-1}$ and $i=40^{\circ}$. Middle right: from the He I $4143 \AA$ line for $T_{\text {eff }}=18250 \mathrm{~K}, \log g=4.1, v_{\Omega}=60 \mathrm{~km} \mathrm{~s}^{-1}$ and $i=30^{\circ}$. Lower left: from the He I $4121 \AA \operatorname{line}$ for $T_{\text {eff }}=18250 \mathrm{~K}, \log g=4.1$, $v_{\Omega}=60 \mathrm{~km} \mathrm{~s}^{-1}$ and $i=30^{\circ}$, taking into account the blends of Fe and O. Lower right: from the Si II 4128-4130 ̊ doublet for $T_{\text {eff }}=18250 \mathrm{~K}$, $\log g=4.1, v_{\Omega}=60 \mathrm{~km} \mathrm{~s}^{-1}$ and $i=30^{\circ}$. For further explanation we refer to the text. 

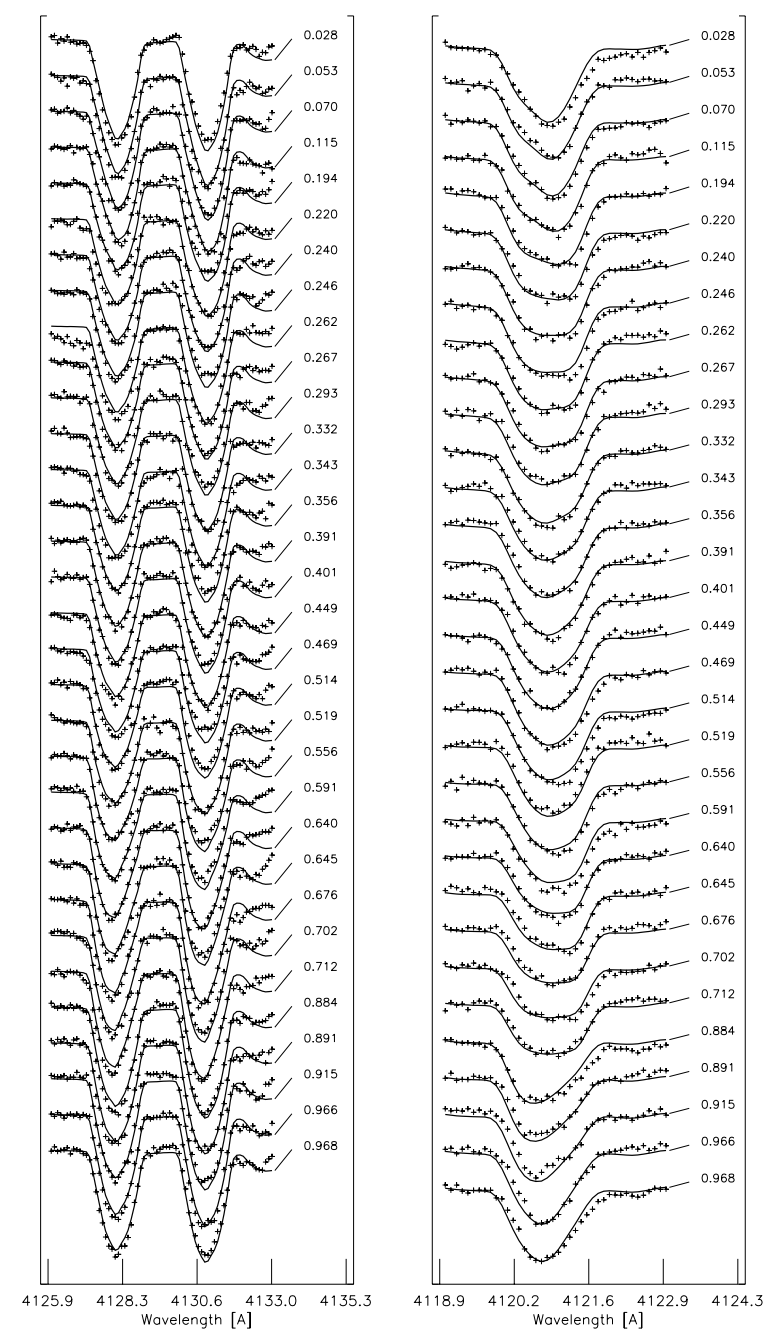

Fig. 7. For HD 131120, observed line profiles (crosses) of the Si II 4128-4130 ̊ lines (left) and of the He I $4121 \AA$ line (right) at different phases of observation and computed line profiles (full lines) obtained with INVERS11.

We varied $v_{\Omega}$ from $70 \mathrm{~km} \mathrm{~s}^{-1}$ to $80 \mathrm{~km} \mathrm{~s}^{-1}$ with a step of $5 \mathrm{~km} \mathrm{~s}^{-1}$ and $i$ from $60^{\circ}$ to $90^{\circ}$ with a step of $10^{\circ}$. The optimum maps were obtained for $v_{\Omega}=75 \mathrm{~km} \mathrm{~s}^{-1}$ and $i=80^{\circ}$. They are shown in Fig. 12 for $\mathrm{Si}$ and He. Figure 13 compares the observed and the calculated $\mathrm{He}$ and $\mathrm{Si}$ profiles at different phases of observation from phase zero (top) to the end of the rotation period (bottom). For silicon, depleted regions compared to the rest of the stellar surface are situated along the equator while enhanced regions are close to the poles. The average abundance of silicon over the stellar surface is almost constant and lower than solar abundance. The mean abundance of helium varies by 0.8 dex on half a period of rotation, which reflects the very large variations of the equivalent width of this line. A strong helium spot is present on the equator surrounded by a very depleted region. For this star, the maps of both elements are very different as already suggested by the very different variations of the moments of the different lines.

\subsection{Summary}

In Table 4 we summarize the physical parameters that we found for the four chemically peculiar B stars from the Doppler mapping. The rotation periods are well determined, which is the case for very few single B stars. The radii are compatible with the values derived from the photometric calibrations listed in Table 3 and are hence an independent spectroscopic confirmation. Equatorial velocities are around $100 \mathrm{~km} \mathrm{~s}^{-1}$. Silicon is found slightly underabundant for the four stars. However their average Si equivalent widths are not too different from the ones of confirmed SPBs (De Cat 2001). Three stars have on average an abundance of helium smaller than the solar one which is -1.05 dex. For HD 131120, helium is underabundant by a factor of 12 and for HD 105382, helium is underabundant by a factor of 4.5. HD 138769 is also He-weak while HD 55522 has on average the solar helium abundance. Future magnetic field measurements on the one hand would be very helpful to understand the surface abundance patterns of our four studied stars. On the other hand, it would be interesting to try and explain our findings for the surface helium and silicon structures from diffusion processes as they are currently understood.

\section{Conclusions}

When the observed variability is monoperiodic, it can be unclear from the analysis of the variations of one single line profile to which cause they must be attributed. However, the comparison of the moment variations of different spectral lines of silicon and helium appeared to be very useful to exclude pulsation as being the cause of the observed monoperiodic variability of the four distinct stars of our sample of candidate SPBs. The peculiarities of these stars compared to confirmed SPBs are the following. The stars appear to be monoperiodic while SPBs of our sample are generally multiperiodic (De Cat 2001; Mathias et al. 2001). The photometry and spectroscopy led to the same period, which is 1.569 days for HD 131120 , 1.295 days for HD 105382, 2.089 days for HD 138769 and 2.769 days for HD 55522. The moments of the studied spectral lines are not sinusoidal but harmonics of the frequency are also present in them. For HD 55522, such a non-sinusoidal signal is also observed in HIPPARCOS and Geneva photometry. The moments computed from the Si II 4128-4130 A doublet and from the He I $4121 \AA$ line do not behave in the same way and are not in phase. The equivalent width variations are large compared to those of SPBs. In particular, the relative $E W$ variation of the He I $4121 \AA$ line for HD 55522 is about $35 \%$ which is remarkably large. All these characteristics cannot be reproduced by pulsation.

In Briquet et al. (2001b) we showed that a good explanation for the variability of the star HD 131120 is its rotation in the presence of non-homogeneous distributions of elements on the stellar surface. As the moments of the line profiles of the three other stars vary in a very similar way as those of HD 131120 , we also interpreted their variability in terms of rotational modulation. For the four stars, we derived stellar surface abundance maps for silicon and helium by means of the Doppler Imaging technique. A future goal is to derive the surface abundance 
Table 4. Physical parameters of our four targets, derived from Doppler mapping.

\begin{tabular}{ccccc}
\hline \hline & HD 131120 & HD 105382 & HD 138769 & HD 55522 \\
\hline$P_{\text {rot }}$ (days) & $1.569 \pm 0.001$ & $1.295 \pm 0.001$ & $2.089 \pm 0.001$ & $2.729 \pm 0.001$ \\
$i$ & $30^{\circ} \pm 10^{\circ}$ & $50^{\circ} \pm 10^{\circ}$ & $40^{\circ} \pm 10^{\circ}$ & $80^{\circ} \pm 10^{\circ}$ \\
$v_{\text {eq }}\left(\mathrm{km} \mathrm{s}^{-1}\right)$ & {$[86 ; 190]$} & {$[75 ; 116]$} & {$[85 ; 150]$} & {$[70 ; 85]$} \\
$R\left(R_{\odot}\right)$ & {$[2.7 ; 5.9]$} & {$[1.9 ; 3.0]$} & {$[3.5 ; 6.2]$} & {$[3.8 ; 4.6]$} \\
Average Si & & & -5.35 & -4.92 \\
abundance (dex) & -4.59 & -4.76 & & \\
$\begin{array}{c}\text { Si abundance } \\
\text { range (dex) }\end{array}$ & {$[-4.53 ;-4.02]$} & {$[-5.36 ;-4.16]$} & {$[-5.70 ;-5.05]$} & {$[-5.29 ;-4.49]$} \\
Average He & & & & -1.06 \\
abundance (dex) & -2.12 & -1.70 & He-weak & \\
He abundance & & & & {$[-2.26 ; 0.46]$} \\
range (dex) & {$[-2.53 ;-1.48]$} & {$[-2.34 ;-0.82]$} & &
\end{tabular}
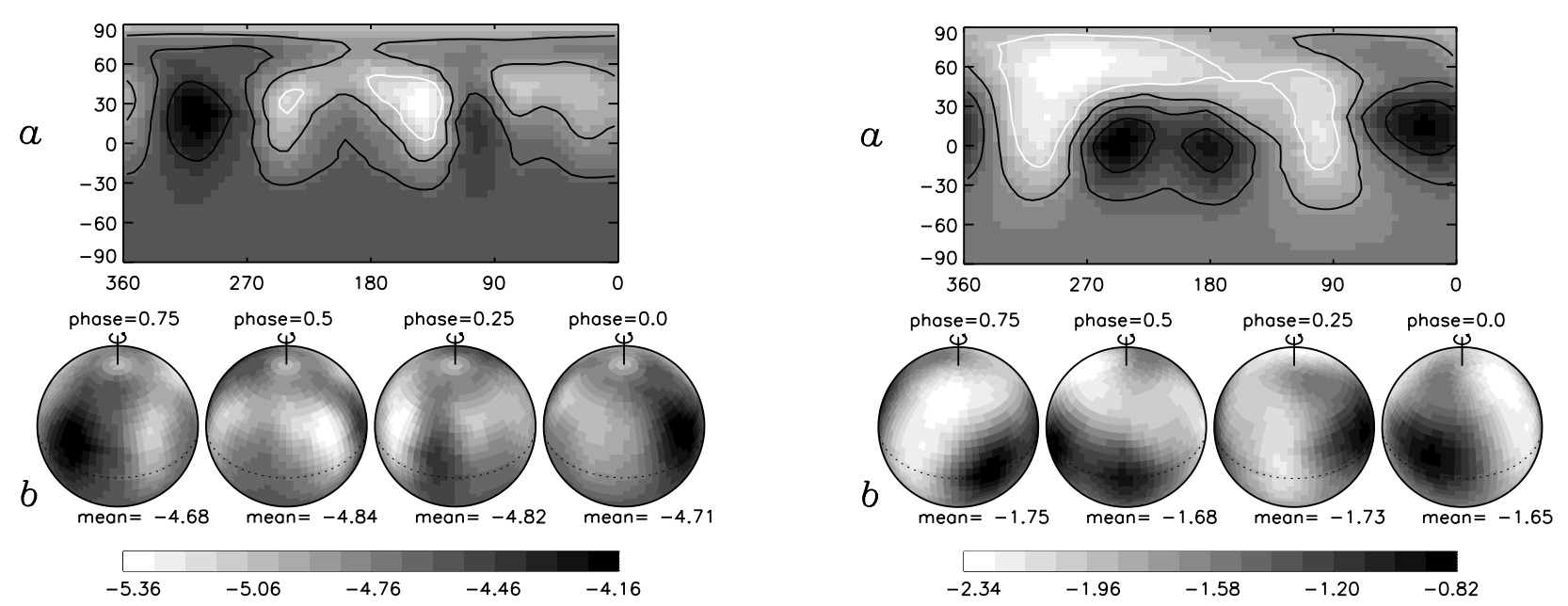

Fig. 8. The silicon distribution (left) and the helium distribution (right) on the surface of HD 105382 obtained with INVERS11 from the Si II 4128-4130 Å doublet and from the He I $4121 \AA$ line for $T_{\text {eff }}=17400 \mathrm{~K}, \log g=4.2, v_{\Omega}=70 \mathrm{~km} \mathrm{~s}^{-1}$ and $i=50^{\circ}$.

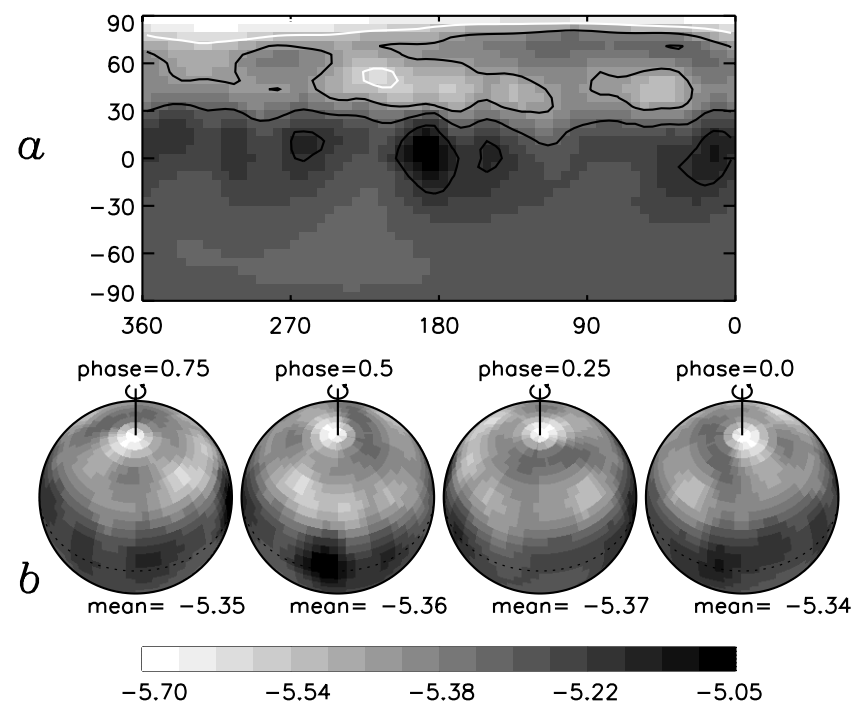

Fig. 10. The silicon distribution on the surface of HD 138769 obtained with INVERS11 from the Si II 4128-4130 A doublet for $T_{\text {eff }}=$ $17500 \mathrm{~K}, \log g=4.2, v_{\Omega}=70 \mathrm{~km} \mathrm{~s}^{-1}$ and $i=40^{\circ}$.

mapping for more elements in these four stars. For HD 131120, we found that helium is significantly depleted over all its stellar surface, which confirms that the star is a He-weak star as already reported in the literature. For this star, regions in which helium is enhanced correspond to regions in which silicon is depleted. This result is also found for HD 105382 that we classify as a new He-weak star since we also found that helium is depleted on average over the whole stellar surface. HD 138769, which is reported as a shell star in the literature, is clearly a Bp He-weak star for which helium is underabundant compared to the Sun but also silicon. For HD 55522, we found the mean abundance value of helium to vary by 0.8 dex during the stellar rotation.

We point out that it is the first time that such detailed spectroscopic analyses and abundance mapping are performed for chemically peculiar B stars with such a high $T_{\text {eff }}$ of about $17500 \mathrm{~K}$. Theoretical diffusion models for such temperatures are needed for a comparison. Moreover, inhomogeneities on the stellar surface are probably correlated to a magnetic field. A search for a magnetic field was only performed for the star HD 131120 but was not conclusive. Such investigations would then be very useful for a better understanding of the surface patterns of these four stars.

Acknowledgements. We thank the referee, Dr. A. P. Hatzes, for his useful suggestions, which helped us to improve this paper. This work was supported by the P.A.I. (Pôle d'Attraction Interuniversitaire) and 

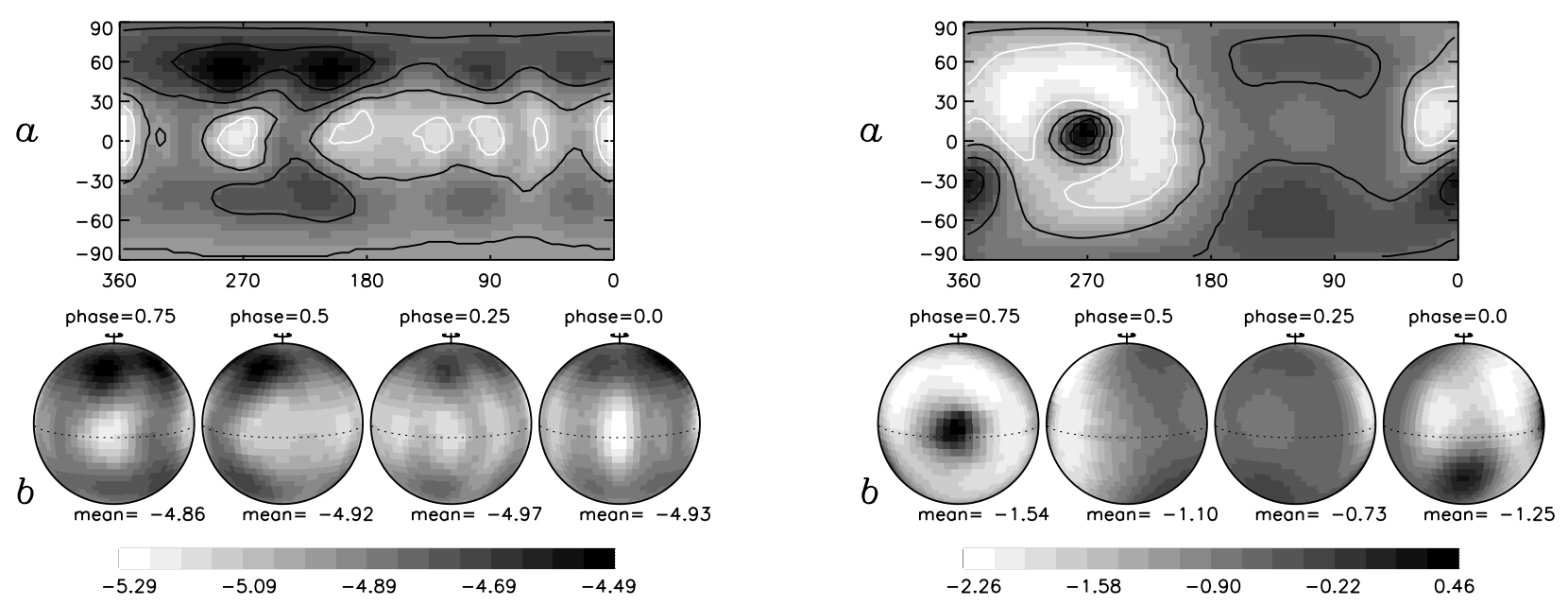

Fig. 12. The silicon distribution (left) and the helium distribution (right) on the surface of HD 55522 obtained with INVERS11 from the Si II 4128-4130 ̊ doublet and from the He I $4121 \AA$ line for $T_{\text {eff }}=17400 \mathrm{~K}, \log g=4.1, v_{\Omega}=75 \mathrm{~km} \mathrm{~s}^{-1}$ and $i=80^{\circ}$.

was carried out within the Belgian Asteroseismology Group. The author from Vienna received funding from the "Fonds zur Förderung der wissenschaftlichen Forschung”, Project P14984.

\section{References}

Aerts, C., De Pauw, M., \& Waelkens, C. 1992, A\&A, 266, 294 Aerts, C., De Cat, P., Peeters, E., et al. 1999, A\&A, 343, 872

Briquet, M., Aerts, C., \& De Cat, P. 2001a, A\&A, 366, 121

Briquet, M., De Cat, P., Aerts, C., \& Scuflaire, R. 2001b, A\&A, 380, 177

De Cat, P. 2001, Ph.D. Thesis, Katholieke Universiteit Leuven, Belgium

de Geus, E. J., de Zeeuw, P. T., \& Lub, J. 1989, A\&A, 216, 44

De Ridder, J., Dupret, M. A., Neuforge, C., \& Aerts, C. 2002, A\&A, 385,572

Flower, P. J. 1996, ApJ, 469, 355

Kochukhov, O., \& Piskunov, N. 2002a, A\&A, 388, 868

Kochukhov, O., Piskunov, N., Ilyin, I., et al. 2002b, A\&A, 389, 420

Künzli, M., North, P., Kurucz, R. L., \& Nicolet, B. 1997, A\&AS, 122, 51

Kupka, F., Piskunov, N. E, Ryabchikova, T. A., et al. 1999, A\&AS, 138,119
Kurucz, R. L. 1992, Stellar Population of Galaxies, ed. B. Barbuy, \& A. Renzini, IAU Symp., 149, 225

Kurucz, R. L. 1993, ATLAS9, Stellar Atmosphere Programs, CDROM No. 13, Smithonian Astrophysical Observatory

Kuschnig, R., Ryabchikova, T. A., Piskunov, N., et al. 1999, A\&A, 348, 924

Lüftinger, T., Kuschnig, R., Piskunov, N., \& Weiss, W. W. 2003, A\&A, accepted

Mathias, P., Aerts, C., Briquet, M., et al. 2001, A\&A, 379, 905

Piskunov, N. E. 1992, in Stellar magnetism, ed. V Yu., I. I. Glagolevskij, Romanyuk (St Petersburg, Nauka), 92

Piskunov, N. E., Kupka, F., Ryabchikova, T. A., et al. 1995, A\&AS, 112,525

Piskunov, N., \& Kochukhov, O. 2002, A\&A, 381, 736

Roberts, D. H., Lehar, J., \& Dreher, J. W. 1987, AJ, 93, 968

Ryabchikova, T. A., Piskunov, N. E., Stempels, H. C., et al. 1999, Physica Scripta, vol. T83, 162

Scargle, J. D. 1981, ApJS, 45, 1

Schaller, G., Schaerer, D., Meynet, G., \& Maeder, A. 1992, A\&A, 96, 269

Stellingwerf, R. F. 1978, ApJ, 224, 953

Waelkens, C., Aerts, C., Kestens, E., et al. 1998, A\&A, 330, 215 
M. Briquet et al.: He and Si surface inhomogeneities of four Bp variable stars, Online Material p 1

\section{Online Material}


M. Briquet et al.: He and Si surface inhomogeneities of four Bp variable stars, Online Material p 2

\section{Appendix A: DI code INVERS11}

The DI code used in this paper was created as a spin-off of a much more complex work on the magnetic DI code INVERS10. INVERS10 was successfully tested and applied to several magnetic CP stars. The description of the code, numerical experiments and applications can be found in Piskunov \& Kochukhov (2002), Kochukhov \& Piskunov (2002a) and Kochukhov et al. (2002b). INVERS11 incorporates the main features of the magnetic code including non-equispaced spatial grid, adaptive wavelength grid, radiative transfer integration combined with the inverse problem solving, implementation of parallel calculations etc. The main differences are:

- magnetic fields are not included in calculations and thus a conventional Feautrier method was selected to compute the emerging spectra;

- since the spectra are re-computed on each iteration there is no fundamental limit on a number of chemical elements mapped simultaneously.

The second property proved to be very useful when dealing with fast rotating stars with rather limited selection of spectral features. Many of these features represent blends of lines of different chemical elements all of which are inhomogeneously distributed over stellar surface (see e.g. Kuschnig et al. 1999). INVERS11 is sufficiently fast to be used on a single or multi-processor workstation and a typical map is produced in a matter of minutes. The input data besides the spectroscopic observations include the projected rotational velocity $v \sin i$, the inclination of rotational axis $i$, spectral resolution of the data, the regularization parameter and the initial guess for the abundances.

The solution of the inverse problem is an iterative process during which the code re-computes synthetic spectra and adjusts the abundance maps in order to obtain the optimal fit to the data. The gradients in the maps are restricted by the regularization function in such a way that the code starts with the lowest spatial frequencies of the solution and gradualy recovers smaller features. The code stops when the data is reproduced within the error bars or when the reproduction of the data cannot be improved.

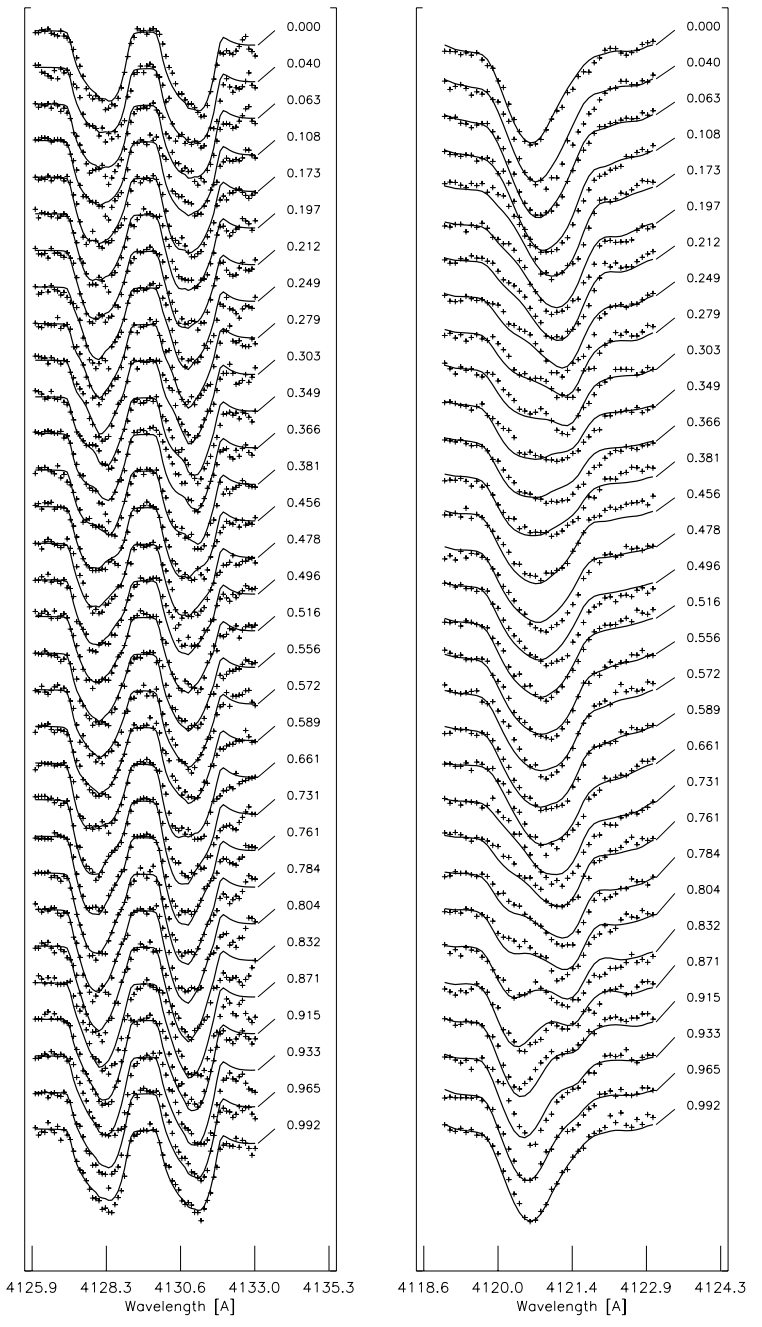

Fig. 9. For HD 105382, observed line profiles (crosses) of the Si II 4128-4130 A lines (left) and of the He I $4121 \AA$ line (right) at different phases of observation and computed line profiles (full lines) obtained with INVERS11. 
M. Briquet et al.: He and Si surface inhomogeneities of four Bp variable stars, Online Material p 3

Table 1. Logbook of the observations of our targets.

\begin{tabular}{ccccccc}
\hline \hline & \multicolumn{3}{c}{ Number of observations } & \multicolumn{3}{c}{ Total time-span (days) } \\
& HIPPARCOS & Geneva & Spectra & HIPPARCOS & Geneva & Spectra \\
\hline HD 105382 & 172 & 132 & 105 & 1173 & 123 & 719 \\
HD 131120 & 53 & 108 & 77 & 861 & 117 & 476 \\
HD 138769 & 83 & 92 & 35 & 1123 & 116 & 691 \\
HD 55522 & 187 & 112 & 56 & 1149 & 417 & 666 \\
\hline
\end{tabular}

Table 2. Observing log for spectroscopy.

\begin{tabular}{|c|c|c|c|c|c|c|c|c|c|c|c|}
\hline \multicolumn{3}{|c|}{ HD 105382} & \multicolumn{3}{|c|}{$\underline{\underline{\text { HD } 131120}}$} & \multicolumn{3}{|c|}{ HD 138769 } & \multicolumn{3}{|c|}{ HD 55522 } \\
\hline \multirow[t]{2}{*}{$\begin{array}{l}\text { Number of } \\
\text { observations }\end{array}$} & \multicolumn{2}{|c|}{$\begin{array}{c}\text { JD } \\
2450000+\end{array}$} & \multirow[t]{2}{*}{$\begin{array}{l}\text { Number of } \\
\text { observations }\end{array}$} & \multicolumn{2}{|c|}{$\begin{array}{c}\text { JD } \\
2450000+\end{array}$} & \multirow[t]{2}{*}{$\begin{array}{l}\text { Number of } \\
\text { observations }\end{array}$} & \multicolumn{2}{|c|}{$\begin{array}{c}\text { JD } \\
2450000+\end{array}$} & \multirow[t]{2}{*}{$\begin{array}{l}\text { Number of } \\
\text { observations }\end{array}$} & \multicolumn{2}{|c|}{$\begin{array}{c}\text { JD } \\
2450000+\end{array}$} \\
\hline & Start & End & & Start & End & & Start & End & & Start & End \\
\hline 44 & 163 & 170 & 27 & 163 & 170 & 9 & 188 & 194 & 6 & 166 & 170 \\
\hline 12 & 188 & 194 & 15 & 188 & 194 & 12 & 272 & 277 & 9 & 188 & 194 \\
\hline 11 & 272 & 277 & 13 & 272 & 277 & 1 & 513 & 513 & 14 & 490 & 498 \\
\hline 10 & 490 & 498 & 4 & 490 & 498 & 1 & 526 & 526 & 8 & 736 & 742 \\
\hline 4 & 513 & 528 & 4 & 513 & 528 & 3 & 571 & 576 & 7 & 779 & 786 \\
\hline 3 & 571 & 576 & 6 & 571 & 576 & 2 & 608 & 608 & 12 & 825 & 831 \\
\hline 1 & 608 & 608 & 8 & 633 & 638 & 6 & 633 & 638 & & & \\
\hline 7 & 633 & 638 & & & & 1 & 878 & 878 & & & \\
\hline 3 & 779 & 786 & & & & & & & & & \\
\hline 8 & 825 & 831 & & & & & & & & & \\
\hline 2 & 882 & 882 & & & & & & & & & \\
\hline
\end{tabular}


M. Briquet et al.: He and Si surface inhomogeneities of four Bp variable stars, Online Material p 4

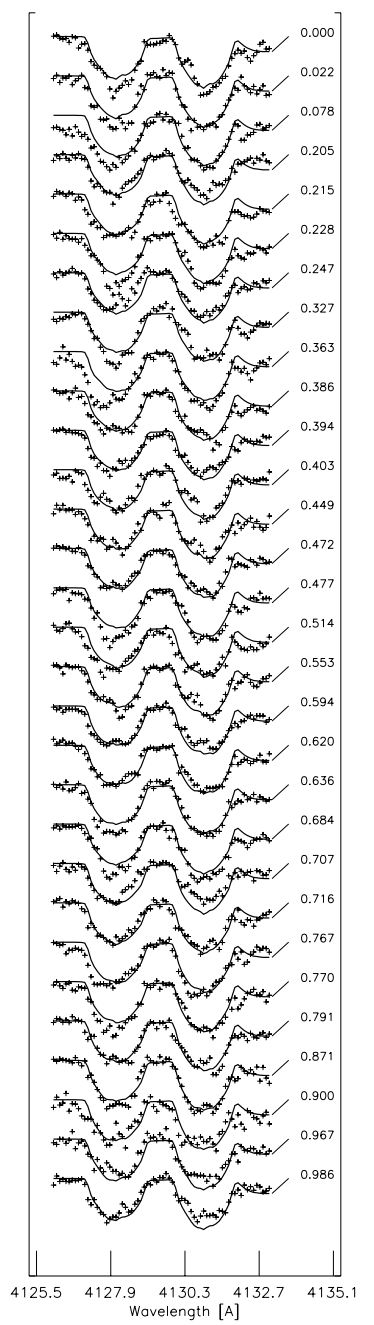

Fig. 11. For HD 138769, observed line profiles (crosses) of the Si II 4128-4130 ̊̊ lines at different phases of observation and computed line profiles (full lines) obtained with INVERS11.
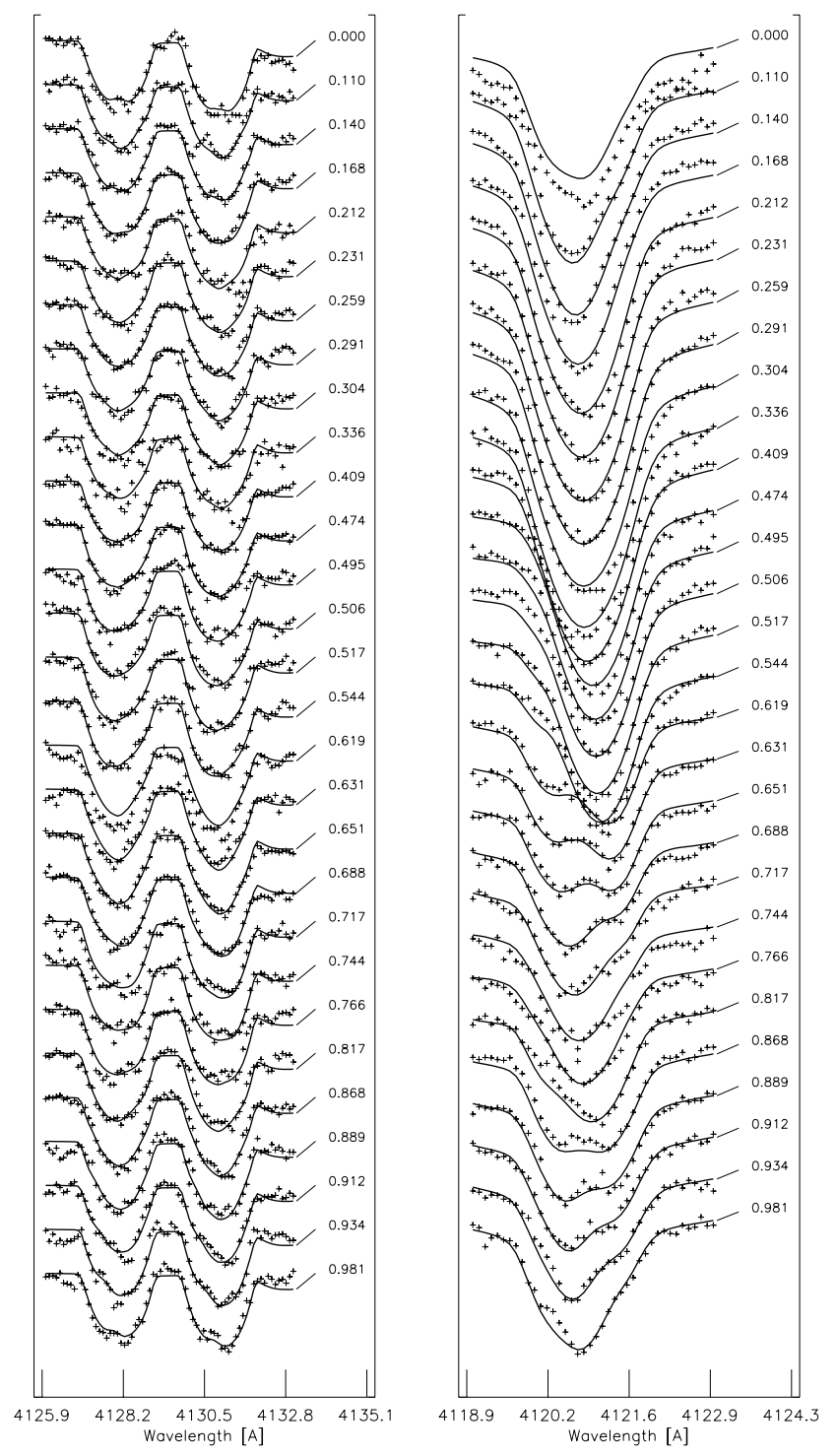

Fig. 13. For HD 55522, observed line profiles (crosses) of the Si II 4128-4130 ̊ lines (left) and of the He I $4121 \AA$ line (right) at different phases of observation and computed line profiles (full lines) obtained with INVERS11. 